\title{
Beta ${ }_{1}$-Adrenergic Receptors Promote Focal Adhesion Signaling Downregulation And Myocyte Apoptosis in Acute Volume Overload
}

\author{
Rachid Seqqat ${ }^{1}$, Xinji Guo ${ }^{1}$, Khadija Rafiq ${ }^{1}$, Mikhail A Kolpakov ${ }^{1}$, Jianfen Guo ${ }^{1}$, Walter J \\ Koch $^{2}$, Steven R Houser ${ }^{1}$, Louis J Dell'italia ${ }^{3}$, and Abdelkarim Sabri ${ }^{1,4}$ \\ ${ }^{1}$ Cardiovascular Research Center and Department of Physiology, Temple University School of \\ Medicine, Philadelphia, PA \\ ${ }^{2}$ Center for Translational Medicine, Temple University School of Medicine, Philadelphia, PA \\ ${ }^{3}$ Department of Medicine, University of Alabama at Birmingham, AL
}

\section{Abstract}

\begin{abstract}
Numerous studies demonstrated increased expression of extracellular matrix (ECM) proteins and activation of focal adhesion (FA) signaling pathways in models of pressure overload-induced cardiac hypertrophy. However, little is known about FA signaling in response to volume overload where cardiac hypertrophy is associated with ECM loss. This study examines the role of beta1adrenergic receptors ( $\beta_{1}$-ARs) in FA signaling changes and myocyte apoptosis induced during acute hemodynamic stress of volume overload. Rats with eccentric cardiac hypertrophy induced after aorto-caval fistula (ACF) develop reduced interstitial collagen content and decreased tyrosine phosphorylation of key FA signaling molecules FAK, Pyk 2 and paxillin along with an increase in cardiac myocyte apoptosis. ACF also increased activation of PTEN, a dual lipid and protein phosphatase, and its interaction with FA proteins. $\beta_{1}$-AR blockade (extended-release of metoprolol succinate, $100 \mathrm{mg}$ QD) markedly attenuated PTEN activation, restored FA signaling and reduced myocyte apoptosis induced by ACF at 2 days, but failed to reduce interstitial collagen loss and left ventricular dilatation. Treating cultured myocytes with $\beta_{1}$-AR agonists or adenoviral expression of $\beta_{1}$-ARs caused PTEN activation and interaction with FA proteins, thus leading to FA signaling downregulation and myocyte apoptosis. Adenoviral-mediated expression of a catalytically inactive PTEN mutant or wild-type FAK restored FA signaling downregulation and attenuated myocyte apoptosis induced by $\beta_{1}$-ARs. Collectively, these data show that $\beta_{1}$-AR stimulation in response to ACF induces FA signaling downregulation through an ECM-independent mechanism. This effect involves PTEN activation and may contribute to adverse cardiac remodeling and function in the course of volume overload.
\end{abstract}

\footnotetext{
(c) 2012 Elsevier Ltd. All rights reserved.

${ }^{4}$ Corresponding author: Abdelkarim Sabri, Ph.D., Cardiovascular Research Center, Temple University, MERB 1045, 3450 N. Broad Street, Philadelphia, PA 19140, Tel. 215-707-4915; Fax. 215-707-5737, sabri@temple.edu.

CONFLICT OF INTEREST

None declared

Publisher's Disclaimer: This is a PDF file of an unedited manuscript that has been accepted for publication. As a service to our customers we are providing this early version of the manuscript. The manuscript will undergo copyediting, typesetting, and review of the resulting proof before it is published in its final citable form. Please note that during the production process errors may be discovered which could affect the content, and all legal disclaimers that apply to the journal pertain.
} 


\section{Keywords}

Volume overload; beta-Adrenergic Receptors; Focal Adhesion; PTEN; Myocyte Apoptosis

\section{INTRODUCTION}

Pathological hemodynamic stress requires increases in sympathetic nervous system activity and $\beta$-adrenergic receptor ( $\beta-A R$ ) activation to maintain cardiac output. However, while sustained $\beta$-AR stimulation is required to maintain cardiac function, it also promotes deleterious changes that contribute to the pathogenesis of heart failure [1]. Treatment of heart failure patients with $\beta$-AR antagonists reduces progression of cardiac dysfunction and mortality [1]. However, the molecular and cellular mechanisms mediating these beneficial effects are not fully understood.

$\beta$-adrenergic receptors belong to the large family of $\mathrm{G}$ protein-coupled receptors that are involved in positive inotropic, chronotropic, and lusitropic responses through activation of $\mathrm{G}$ stimulatory protein (Gs) [2]. Transgenic mice expressing supraphysiological levels of cardiac $\beta_{1}$-AR [3], $\beta_{2}$-AR [4], or Gs [5] develop reduced cardiac function, enhanced fibrosis, apoptosis, and myocyte hypertrophy. Increased myocyte apoptosis is thought to be exclusively mediated by the $\beta_{1}$-AR subtype, in-vitro[6] and in-vivo [7]. This $\beta_{1}$-ARstimulated pro-apoptotic effect appears to be blocked by protein kinase A (PKA) inhibition in adult rat myocytes [8]. Recently, integrin signaling has been proposed as a novel mechanism by which $\beta_{1}$-ARs induce myocyte apoptosis [9]. The molecular mechanisms by which $\beta_{1}$-AR stimulation regulates integrin signaling remain largely unknown.

Myocyte adhesion to extracellular matrix (ECM) through integrin receptors is critical for normal cardiac function [10,11]. Perturbation of proper integrin-ECM interactions is observed in various acute and chronic cardiac pathological conditions including pressure overload and ischemia/reperfusion injury [10]. Loss of such interactions in normal cells results in the onset of apoptosis referred to as anoikis [11]. Binding of a matrix protein to an integrin heterodimer typically results in the activation of the non-receptor tyrosine kinase FAK, which in turn recruits Src, p130 Cas , paxillin and other signaling intermediates [10,11]. Integrin $\beta 1$ or FAK knockout results in defective heart development and early embryonic lethality $[12,13]$. Interestingly, myocyte-restricted deletion of the $\beta 1$-integrin in adult mouse hearts leads to a dilated cardiomyopathy and concomitant heart failure[14] whereas myocyte-restricted FAK inactivation leads to the development of eccentric cardiac hypertrophy with age and in response to pressure overload stimuli [15]. Interestingly, persistent challenge of mice with myocyte-restricted FAK inactivation leads to enhanced cardiac dysfunction in comparison with challenged controls [16]. Thus, the coordinated activation of integrin and focal adhesion (FA) signaling is likely to be critical in diverse cellular processes such as myofibril organization, myocyte hypertrophy and apoptosis.

Changes in FA signaling in the heart have been shown in models of pressure overloadinduced cardiac hypertrophy where increased expression of ECM and integrins associated with activation of FA signaling pathways correlate with increased cardiac hypertrophy [17, 18]. We recently showed that early induction of volume overload (VO) after mitral regurgitation in dogs decreases FA signaling [19]. The present study support the hypothesis that in VO, $\beta_{1}$-AR-mediated activation of lipid and protein phosphatase PTEN (Phosphatase and Tensin homologue on chromosome Ten) leads to FA signaling downregulation and myocyte apoptosis. These changes appear to be independent of associated reductions in ECM and may contribute to adverse left ventricular (LV) remodeling in response to VO. 


\section{METHODS}

\subsection{Animal Preparation}

All animal protocols have been approved by the Institutional Animal Care Committee of Temple University and Investigations were conformed to the Guide for the Care and Use of Laboratory Animals published by the US National Institutes of Health and approved by the American Physiological Society. Male Sprague-Dawley rats (250-300g) were anaesthetized with a mixture of xylazine $(10 \mathrm{mg} / \mathrm{kg}$, i.p. $)$ and ketamine $(80 \mathrm{mg} / \mathrm{kg}$, i.p.). Once deep anesthesia was confirmed by the absence of toe pinch reflex, an infrarenal abdominal aortato-vena cava fistula (ACF) was created as we previously described [20]. Age-matched shamand ACF-operated rats were generated for study at 6-hrs, 12-hrs, 2-days and 5-days ( $\mathrm{n}=6$ per group). In a third group of animals, sham and ACF operated rats were treated for 2-days with vehicle or $\beta_{1}-\mathrm{AR}$ blocker (extended-release of metoprolol succinate, $100 \mathrm{mg} / \mathrm{kg} / \mathrm{d} \mathrm{PO}$ ) ( $\mathrm{n}=6$ per group) starting 30-min after ACF induction. After each time, animals were sacrificed and hearts were either arrested in diastole for histology or snap frozen in liquid nitrogen for molecular and enzyme activity assays analysis.

\subsection{Hemodynamic and Echocardiographic Measurements}

Rats were anesthetized with ketamine $(80 \mathrm{mg} / \mathrm{kg}$, i.p.) and xylazine $(10 \mathrm{mg} / \mathrm{kg}$, i.p.). Highfidelity LV pressure (SPR-249A Millar Mikro-Tip catheter transducer, Millar Instruments) was recorded concurrent with echocardiography (Agilent Sonos-5500, Philips). LV function was calculated as described previously [20].

\subsection{Histology}

The heart was arrested in diastole, perfused fixed, dehydrated, and embedded in paraffin. Sections were stained with Sirius-red reagent for detecting collagen. Details of procedures for collagen staining and quantification are provided in the Supplementary material.

\subsection{Neonatal rat cardiomyocyte (NRCM) isolation}

Two-day old Sprague-Dawley rats were subjected to hypothermic cardiac arrest, followed by decapitation. After removal of the heart, myocytes were isolated from the ventricles by collagenase digestion as previously described [20] with additional details provided in the Supplementary material.

\subsection{Western blotting and Immunoprecipitation assays}

Western blotting and immunoprecipitation assays were performed using standard techniques and described in details the Supplementary material.

\subsection{Assessment of Apoptosis}

Apoptosis was assessed by terminal deoxytransferase-mediated dUTP nick-end labelling (TUNEL), by measuring the cytosolic histone-associated mono- and oligo-nucleosomes fragments associated with apoptotic cell death, and by measuring caspase- 3 activity. The Details of procedures for assessing apoptosis were described in details in the Supplementary material.

\subsection{Statistical Analyses}

Data reported are mean \pm SEM. Statistical significance was evaluated using ANOVA posthoc test. A $P$ value less than 0.05 was considered significant.

An expanded Materials and Methods section is in the online-only Data Supplement. 


\section{RESULTS}

\subsection{ACF induces eccentric LV hypertrophy and decreases interstitial collagen}

ACF caused LV dilatation with significant increases in LV end-diastolic dimension (LVEDD) at 2- and 5-days (Table 1). The ratio of LVEDD to LV posterior wall thickness (LVEDD/LVPWT) also increased at 2- and 5-days after ACF. LV end-systolic dimension (LVESD) was similar in both ACF and sham groups while LV ejection fraction increased significantly at 2- and 5-days after ACF compared to sham groups.

Mean arterial pressure was significantly decreased at 6- and 12-hrs post-ACF compared to shams, and returned towards shams at 2 - and 5-days after ACF ( $<<0.05$ vs. sham). Mean heart rate significantly increased at 6-hrs after $\mathrm{ACF}$ and remained elevated throughout the time of the study. No significant change in LV end systolic pressure (LVESP) was detected between ACF and sham groups at any time studied. In contrast, LV end-diastolic pressure (LVEDP) was slightly increased at 2 - and 5-days post ACF ( $<<0.05$ vs. sham).

There was a significant increase in heart weight (HW) to body weight (BW) ratio at 2-days and 5-days after ACF compared to sham controls ( $\mathrm{p}<0.05$ vs. shams). The increase in HW resulted from an increase in both left and right ventricular weights (data not shown) without any significant difference in BW between the control and fistula groups. There was a significant decrease in interstitial collagen volume percent at 12-hrs after ACF that persisted at day 2 , and returned toward sham values by 5 -days after ACF (Figure 1A).

\subsection{Impaired FA signaling during acute ACF in rats}

In animal models of pressure overload, cardiac hypertrophy is associated with increased ECM deposition and FA signaling activation [10]. However, little is known about FA signaling in the eccentric cardiac hypertrophy of VO where there is a decrease in ECM deposition [19,21,22]. We determined if critical components of the FA signaling cascade were altered after ACF. Specifically, we examined tyrosine phosphorylation of FA proteins FAK at $\mathrm{Y}^{397}$, a site that is phosphorylated upon ECM binding to integrins and is necessary for FAK activation $[10,11]$. ACF significantly decreased $\mathrm{Y}^{397}$-FAK phosphorylation at 2and 5-days compared to shams (Figure 1B). This decrease did not result from a change in FAK accumulation as equal FAK protein levels were found in sham and ACF LV lysates. Similar decreases in tyrosine phosphorylation of $\mathrm{Pyk}_{2}$ (a FAK related tyrosine kinase) and paxillin (a cytoskeletal kinase associated with FAK) were also observed at 2-days after ACF compared to shams (Figure 1C) with no change in $\mathrm{Pyk}_{2}$ or paxillin accumulation between sham and ACF animals. Consistent with a reduction in FAK tyrosine phosphorylation, ACF decreased FAK interaction with p130 Cas and paxillin, two important components of FA that associate with and are phosphorylated by FAK (Figure 1D).

\section{3. $\beta_{1}-A R$ blockade $\left(\beta_{1}-R B\right)$ attenuates $F A$ signaling downregulation post-ACF without affecting collagen accumulation}

Changes in ECM and FA signaling pathways occurred rapidly after the induction of VO. Therefore, we next explored the role of increased $\beta_{1}$-AR activation in triggering these changes. Treatment with metoprolol, a selective $\beta_{1}$-AR blocker, for two days had very little effect on heart rate in sham operated animals, but significantly reduced the increase in heart rate induced by ACF (Supplemental Table 1). $\beta_{1}$-RB did not prevent the increase in ejection fraction, fractional shortening, and LVEDD induced by ACF (Figure 2A and Supplemental Figures S1). However, $\beta_{1}-\mathrm{RB}$ significantly attenuated the increase in LVEDD to LV wall thickness ratio induced by ACF and this effect resulted mainly from increased LV wall thickness (Supplemental Figure S1). The $\beta_{1}-\mathrm{RB}$ did not alter the HW/BW ratio or the 
reduction in collagen volume percent induced after ACF (Figures 2B and 2C). Interestingly, $\beta_{1}$-RB markedly attenuated $\mathrm{Y}^{397}$-FAK dephosphorylation induced by ACF (Figures 2D).

\section{4. $\boldsymbol{\beta}_{1}-\mathrm{RB}$ prevents PTEN activation induced by acute ACF}

To determine the link between $\beta_{1}$-AR stimulation and $Y^{397}$-FAK dephosphorylation, we evaluated several regulatory mechanisms involved in FAK phosphorylation at the $\mathrm{Y}^{397}$ residues, including FAK phosphorylation at $\mathrm{Y}^{407}$, FAK phosphorylation at $\mathrm{S}^{910}$ or increased expression of FAK Related Non-Kinase (FRNK), a 42-kDa protein whose amino acid sequence is identical to the non-catalytic carboxyl-terminal sequence of FAK and acts as a dominant-interfering mutant of FAK [11]. There were no detectable changes in the accumulation of FRNK, $\mathrm{Y}^{407}$-FAK, or $\mathrm{S}^{910}$-FAK between sham and ACF either untreated or treated with $\beta_{1}-\mathrm{RB}$ (Supplemental Figure S2).

FAK phosphorylation at $\mathrm{Y}^{397}$ residues can also be regulated by protein tyrosine phosphatases. Several phosphatases including $\mathrm{SHP}_{2}$ and PTEN have been shown to decrease FAK tyrosine phosphorylation [23,24]. LV lysates analysis shows that ACF had no detectable effect on $\mathrm{SHP}_{2}$ phosphorylation. However, there was a significant increase in non-phospho PTEN accumulation, indicative of PTEN dephosphorylation and activation (Figures 2E) [24]. No change in PTEN expression was observed in ACF or ACF $+\beta_{1}-\mathrm{RB}$ groups compared to shams. The increase in non-phospho PTEN accumulation was associated with an increase in PTEN interaction with FAK (Figure 2F). $\beta_{1}$-RB markedly inhibited PTEN activation and the increase in PTEN/FAK interaction induced by ACF.

\section{5. $\beta_{1}$-RB prevents myocyte apoptosis induced by acute ACF}

Both loss of ECM and/or loss of FA signaling have been shown to promote cell apoptosis $[10,11,23]$. To confirm that apoptosis occurs after FA signaling alterations during ACF, we measured DNA fragmentation and caspase-3 activity in LV of sham and ACF animals. As we have shown previously [20], TUNEL-positive myocytes were found in shams and significantly more were observed 2-days after ACF (Figure 3A). Treatment with $\beta_{1}$-RB significantly attenuated the number of TUNEL-positive myocytes induced by ACF. DNA fragmentation, as assessed by ELISA, and caspase-3 activity were also significantly increased in ACF LV homogenates and $\beta_{1}$-RB significantly attenuated this increase (Figures $3 \mathrm{~B}$ and $3 \mathrm{C}$ ). These results show that $\beta_{1}-\mathrm{RB}$ reduces myocyte apoptosis in response to acute VO.

We next examined the molecular mechanisms responsible for myocyte protection mediated by $\beta_{1}-\mathrm{RB}$. Relative to sham controls, ACF heart samples showed increases in the levels of p38 MAPK and JNK phosphorylation, two stress activated protein kinases known to be involved in myocyte apoptosis induced by $\beta$-AR stimulation (Figure 3D) [25, 26]. In contrast, $\mathrm{ACF}$ also increased AKT phosphorylation and $\mathrm{Bcl} 2$ expression, two signaling molecules that are cardioprotective. $\beta_{1}$-RB markedly attenuated ACF-induced changes in all of these pro- and anti-apoptotic signaling molecules, with the net result being reduced myocyte apoptosis.

\subsection{Prolonged $\beta_{1}$-AR stimulation induces FA signaling downregulation in isolated myocytes}

To further explore the role of $\beta_{1}$-AR-dependent alteration of FA signaling observed after acute ACF in cardiomyocytes, we examined the effect of $\beta_{1}$ - and $\beta_{2}$-AR stimulation on FA signaling in cultured neonatal rat cardiomyocytes. Upon treatment with isoproterenol (ISO), a non selective $\beta_{1}$ - and $\beta_{2}$-AR agonist, FAK was rapidly tyrosine phosphorylated, reaching a maximum within 5-10 min (Figure 4A). The increase in FAK phosphorylation was associated with an increase in its interaction with $\mathrm{p} 130^{\mathrm{Cas}}$ and paxillin (Figure 4B). Similar 
increases in FAK tyrosine phosphorylation and association with $\mathrm{p} 130^{\mathrm{Cas}}$ and paxillin were observed after treatment with norepinephrine (NE, a non selective $\alpha$ - and $\beta$-AR agonist) in the presence of prazosin (a selective a-AR antagonist). Pretreatment of cells with a selective $\beta_{1}$-AR antagonist, CGP0712A, attenuated FAK tyrosine phosphorylation and its association with $\mathrm{p} 130^{\mathrm{Cas}}$ and paxillin induced by ISO treatment for 5 -min. Treatment of cells with zinterol, a selective $\beta_{2}$-AR agonist, slightly increased FAK tyrosine phosphorylation and its association with $\mathrm{p} 130^{\mathrm{Cas}}$ and paxillin. Collectively, these data show that acute stimulation of $\beta_{1}$-ARs, and to slight degree of $\beta_{2}$-ARs, increases FA signaling.

In contrast to acute $\beta_{1}$-AR stimulation, sustained treatment with ISO for 12-, 24-, or 48-hrs significantly decreased tyrosine phosphorylation of FAK, Pyk $_{2}$, and Paxillin with no effect on their expression levels (Figure 4C). ISO or NE+prazosin treatment for 24-hrs also decreased association between FAK and $\mathrm{p} 130^{\mathrm{Cas}}$ or FAK and paxillin (Figure 4D). This effect on FA signaling downregulation was mediated through $\beta_{1}$-AR stimulation, as pretreatment with CGP0712A reduced ISO-induced FAK tyrosine dephosphorylation and its dissociation from $\mathrm{p} 130^{\mathrm{Cas}}$ and paxillin, while treatment with zinterol had no detectable effect on FAK tyrosine phosphorylation and FAK dissociation from $\mathrm{p} 130^{\mathrm{Cas}}$ and paxillin.

To independently confirm the role of $\beta_{1}$-AR stimulation on FA signaling downregulation, NRCMs were infected with adenovirus carrying wild type (WT) $\beta_{1}$-AR. Overexpression of $\beta_{1}$-ARs dose dependently decreased FAK, Pyk 2 , and paxillin phosphorylation and attenuated FAK interaction with $\mathrm{p} 130^{\mathrm{Cas}}$ and paxillin. It is noteworthy that high titers of $\beta_{1}$-AR adenoviruses were associated with FAK, Pyk 2 and paxillin degradation and myocyte death (Figures $4 \mathrm{E}$ and $4 \mathrm{~F}$ and data not shown). These data demonstrate that acute $\beta_{1}$-AR stimulation leads to FA signaling activation while persistent stimulation of these receptors leads to FA signaling downregulation.

\section{7. $\beta_{1}$-AR stimulation induces PTEN activation and its interaction with FAK}

The fact that PTEN activation and FA signaling downregulation post-ACF were abolished by $\beta_{1}-\mathrm{RB}$, we next assessed the role of $\beta_{1}$-AR stimulation on PTEN activation. ISO or NE +Prazosin treatments for 12- and 24-hrs increased significantly non-phospho PTEN accumulation and PTEN interaction with FAK (Figures 5A and 5B). $\beta_{1}$-AR adenoviral expression also dose dependently increased non-phospho PTEN accumulation compared to Lac-Z infected controls demonstrating the role of $\beta_{1}$-AR stimulation in mediating PTEN activation (Figure 5C)

We further explored the mechanisms by which $\beta_{1}$-AR induced non-phospho PTEN accumulation. Induction of cAMP production in response to the adenylate cyclase agonist forskolin significantly increased non-phospho PTEN accumulation and subsequent $\mathrm{Y}^{397}$ FAK dephosphorylation (Figure 5A). Moreover, pretreatment with BAPTA, a Ca ${ }^{++}$chelator, or KN93, a $\mathrm{Ca}^{2+} /$ calmodulin-dependent protein kinase II (CaMKII) inhibitor, significantly attenuated non-phospho PTEN accumulation and subsequent $\mathrm{Y}^{397}$-FAK dephosphorylation induced by $\beta_{1}$-AR stimulation (Figure 5D). These data suggest that cAMP/Ca ${ }^{++} / \mathrm{CaMKII}$ signaling mediates PTEN activation induced by persistent stimulation of $\beta_{1}$-ARs.

\subsection{Inhibition of PTEN prevents FA signaling alteration and myocyte apoptosis induced by $\beta_{1}$-AR stimulation}

To determine the role of PTEN in FA signaling downregulation induced by $\beta_{1}$-AR stimulation, NRCMs were infected with either WT-PTEN, dominant negative (DN)-PTEN (a catalytically inactive PTEN in which cysteine 124 has been mutated to serine (C/S)) or Lac-Z. Overexpression of WT-PTEN decreased basal FAK and paxillin tyrosine phosphorylation compared to Lac-Z infected controls and treatment with ISO further 
enhanced this decrease (Figure 6A). In contrast, overexpression of DN-PTEN prevented FAK and paxillin tyrosine dephosphorylation induced by ISO.

To confirm the role of PTEN in $\beta_{1}$-AR-induced myocyte apoptosis, we conducted gain- or loss-of-function experiments, in which we transduced NRCMs with adenoviruses carrying WT-PTEN, DN-PTEN, or Lac-Z. ISO treatment induced an increase in caspase-3 activity and DNA fragmentation indicative of myocyte apoptosis (Figures 6B and 6C).

Overexpression of DN-PTEN had no effect on basal myocyte apoptosis compared to control Lac-Z infected cells, but prevented myocyte apoptosis induced by ISO. In contrast, WTPTEN overexpression increased basal myocyte apoptosis compared to control Lac-Z infected myocytes and enhanced myocyte apoptosis in response to ISO treatment. Overexpression of WT-FAK also significantly attenuated caspase-3 activation and DNA fragmentation induced by ISO treatment, while expression of $\mathrm{Y}^{397}$-FAK, a mutant of FAK that lacks the autophosphorylation site of FAK that is necessary for its activity, significantly increased basal myocyte apoptosis and enhanced ISO-induced caspase-3 activation and DNA fragmentation (Figures 6D and 6E). These data show that sustained stimulation of $\beta_{1^{-}}$ ARs mediates PTEN activation that causes FA signaling downregulation and subsequent myocyte apoptosis.

\section{DISCUSSION}

The results of this study reveal important roles of $\beta_{1}$-AR stimulation in mediating FA signaling downregulation and myocyte apoptosis during the acute phase of VO. The absence of $\beta_{1}-\mathrm{RB}$ effects on LV remodeling or interstitial collagen loss with acute ACF rules out any involvement of mechanical factors or ECM proteins in regulating FA signaling alterations induced by acute ACF. Instead, our in-vitro studies provide evidence that sustained stimulation of $\beta_{1}$-ARs leads to FA signaling downregulation and myocyte apoptosis through activation of PTEN, suggestive of an important role of this phosphatase in modulating $\beta_{1^{-}}$ AR-induced responses in cardiomyocytes (Supplemental Figure S3).

Our results demonstrate that FAK tyrosine phosphorylation and its interaction with p130 Cas and paxillin, two important components of FA complex that associate with and are phosphorylated by FAK $[10,11]$, were significantly altered after the acute stress of ACF. This effect on FAK phosphorylation was also observed for $\mathrm{Pyk}_{2}$ and paxillin tyrosine phosphorylation indicating a general downregulation of FA signaling as well as destruction of the FA complex. Interestingly, the decrease in FAK, $\mathrm{Pyk}_{2}$ and paxillin tyrosine phosphorylation occurred despite a lack of changes in $\beta_{1}$-integrin expression (data not shown) and in the absence of cleavage of these molecules as was observed in the failing and dilated hearts [27]. This may be due to differences in the amount of caspase- 3 activity between acute ACF and dilated cardiomyopathy or to the presence of other factors that may protect FA molecules from caspase-3 cleavage during the acute phase of ACF. However, these findings in VO hearts are in stark contrast to the activation of FA signaling reported in the early compensated stages of experimentally-induced pressure overload in-vivo or in isolated cardiomyocytes subjected to pulsatile mechanical stretch $[17,18]$. Similar trends in FA signaling were also observed in a model of VO induced by acute mitral regurgitation in dogs[19] suggesting that FA signaling downregulation characterizes hearts with eccentric hypertrophy and that factors other than mechanical stimuli mediate FA signaling alterations during acute ACF.

Using $\beta_{1}$-RB therapy, we provided evidence that $\beta_{1}$-AR stimulation mediates FA signaling alterations during the acute phase of ACF. This effect occurred in the absence of $\beta_{1}-\mathrm{RB}$ effect to restore interstitial collagen loss and to attenuate $\mathrm{LV}$ remodeling associated with $\mathrm{ACF}$, suggesting that intrinsic stimulation of $\beta_{1}$-ARs is the main trigger of FA signaling 
alterations induced after ACF. These data are consistent with previous studies showing the role of $\beta_{1}-\mathrm{RB}$ therapy in increasing myofibrillar content, restoring $\mathrm{Ca}^{2+}$ transients, and improving fractional shortening in cardiomyocytes isolated from mitral regurgitation hearts compared to shams without affecting interstitial collagen accumulation [22]. Moreover, $\beta_{1^{-}}$ $\mathrm{RB}$ has been shown to restore $\mathrm{Ca}^{2+}$ handling and to improve $\mathrm{Ca}^{2+}$ transients in cardiomyocytes from human failing hearts [28]. The discordance between FA signaling findings and LV remodeling could be explained by the failure of $\beta_{1}-\mathrm{RB}$ to attenuate the loss of interstitial collagen as has been shown in dogs with mitral regurgitation treated with $\beta_{1^{-}}$ $\mathrm{RB}[19,21]$. Therefore, our data suggest a role for ECM/cell interaction in mediating LV dilatation and a role for $\beta_{1}$-AR-induced FA signaling alterations in mediating myocyte apoptosis in response to ACF.

Our present findings show that activation of PTEN, a lipid and protein phosphatase known to mediate FAK and p130 Cas tyrosine dephosphorylation [24], mediates FA signaling alterations in response to sustained $\beta_{1}$-AR stimulation. PTEN activation and interaction with FAK were markedly increased after ACF and these effects were attenuated in animals treated with $\beta_{1}-\mathrm{RB}$. Consistent with these findings in-vivo, we found that persistent stimulation of $\beta_{1}$-ARs increased PTEN activation and its interaction with FAK in cultured myocytes. Moreover, the $\beta_{1}$-AR pathway for activation of PTEN involves elevation of intracellular $\mathrm{Ca}^{++}$and cAMP levels and activation of the CaMKII pathway. These data are consistent with the findings showing the role of increased cAMP levels in PTEN activation or $\mathrm{Y}^{397}$-FAK dephosphorylation in other cell types $[29,30]$. Intriguingly, loss of PTEN activity was sufficient to restore FA signaling alterations and to attenuate myocyte apoptosis induced after $\beta_{1}$-AR stimulation. PTEN expression has been shown to be increased in hearts of animals treated with isoproterenol[31] and its inactivation induces cardiomyocyte hypertrophy both in-vitro[31] and in-vivo[32] and suppresses the development of pathological cardiac hypertrophy in response to biomechanical stress [33]. However, PTEN functions primarily as a lipid phosphatase that lowers the levels of the $\mathrm{PI}_{3} \mathrm{~K}$ product PIP3 within the cells and antagonizes $\mathrm{PI}_{3} \mathrm{~K}$-mediated cellular signaling [24]. Therefore, PTEN activation in response to $\beta_{1}$-AR stimulation may regulate crucial signal transduction pathways other than FA signaling cascade. It remains to be determined whether PTEN might contribute to changes in cardiac remodeling and function in response to ACF.

The role of the constitutive phosphorylation of FA proteins in normal cellular function is not fully understood, but may be important for maintaining cell survival signaling and FA integrity in the resting state [10,11]. Previously, we reported that acute ACF induces myocyte apoptosis that was associated with cardiac dilatation [20]. The results of the present work further substantiated the role of $\beta_{1}$-AR stimulation as a mediator of this myocyte apoptosis based on the finding that myocyte apoptosis was abolished in ACF animals treated with $\beta_{1}$-AR blockers. Using gain or loss-of-function of PTEN and FAK, we provided evidence that PTEN activation and FAK signaling downregulation mediate myocyte apoptosis induced by $\beta_{1}$-AR stimulation. We also found that activation of the PTEN/FAK pathway involves $\beta_{1}$-AR-induced $\mathrm{cAMP} / \mathrm{Ca}^{++}$elevation and CaMKII activation, suggesting that this pathway could represent a potential target for $\mathrm{cAMP} / \mathrm{Ca}^{++} / \mathrm{CaMKII}$ in mediating $\beta_{1^{-}}$ AR-induced myocyte apoptosis as has been described in previous studies [8,34]. Disruption of FAK signaling has been shown to prevent myocyte hypertrophy and to promote myocyte death in-vitro $[23,35,36]$. These findings in-vitro were further corroborated in mice with conditional cardiomyocyte-specific deletion of FAK where persistent challenge of these mice with pressure overload enhanced myocyte apoptosis and cardiac dysfunction in comparison with challenged genetic controls [16]. Collectively, these findings show the link between FAK signaling alterations and the development of eccentric cardiac hypertrophy, myocyte apoptosis and heart failure. 
The selective down-regulation of FA signaling in the acute phase of ACF was associated by a small amount of LV dilatation and increased LV fractional shortening. This occurs in spite of activation of the renin-angiotensin-system and adrenergic drive and is in stark contrast to the activation of FA signaling in experimentally-induced pressure overload in-vivo [17,37]. In these ACF animals, $\beta_{1}-\mathrm{RB}$ decreased catecholamine-induced cardiac response to ACF and restored FA signaling without affecting LV dilatation. In addition, $\beta_{1}$-RB did not prevent a marked loss of interstitial collagen, which could result in a better short-term diastolic remodeling and function. It is important to note that this study only addressed the acute stage of VO. Whether FA signaling alterations occur in the chronic phases of VO and whether $\beta_{1}-\mathrm{RB}$ restores these alterations must be addressed in future studies. Long-term therapy of $\mathrm{VO}$ with $\beta_{1}$-RB has been shown to improve isolated cardiomyocyte function but has no effect on interstitial collagen loss and LV dilatation and remodeling [22].

In conclusion, our data suggest that the primary activation of PTEN and subsequent loss of FA signaling offers a new target, in addition to $\beta_{1}$-ARs, to attenuate excessive adrenergic drive in the VO. This loss of FA signaling, along with cell-ECM network disruption, raises the intriguing possibility that $\beta_{1}$-AR stimulation of PTEN and subsequent alteration in FA signaling may contribute to adverse cardiac remodeling and function in the course of VO.

\section{Supplementary Material}

Refer to Web version on PubMed Central for supplementary material.

\section{Acknowledgments}

\section{FUNDING SOURCES}

This work was supported by the National Institute of Health (HL360338, HL 360343).

We thank Dr. Steven Phillips for his critical reading of the manuscript.

\section{Non-standard Abbreviations}

$\begin{array}{ll}\beta \text {-AR } & \beta \text {-adrenergic receptor } \\ \text { ECM } & \text { extracellular matrix } \\ \text { FA } & \text { focal adhesion } \\ \text { VO } & \text { volume overload } \\ \text { LV } & \text { left ventricular } \\ \text { ACF } & \text { abdominal aorta-to-vena cava fistula } \\ \text { NRCM } & \text { neonatal rat cardiomyocytes } \\ \beta_{1}-\mathbf{R B} & \beta_{1} \text {-AR blockade } \\ \text { ISO } & \text { isoproterenol } \\ \text { NE } & \text { norepinephrine }\end{array}$

\section{References}

1. Port JD, Bristow MR. Altered beta-adrenergic receptor gene regulation and signaling in chronic heart failure. J Mol Cell Cardiol. 2001; 33(5):887-905. [PubMed: 11343413]

2. Steinberg SF. The molecular basis for distinct $\{$ beta $\}$-adrenergic receptor subtype actions in cardiomyocytes. Circ Res. 1999; 85(11):1101-11. [PubMed: 10571542] 
3. Engelhardt S, Hein L, Wiesmann F, Lohse MJ. Progressive hypertrophy and heart failure in beta 1adrenergic receptor transgenic mice. Proc Nat Acad Sci USA. 1999; 96(12):7059-64. [PubMed: 10359838]

4. Liggett SB, Tepe NM, Lorenz JN, Canning AM, Jantz TD, Mitarai S, et al. Early and delayed consequences of beta(2)-adrenergic receptor overexpression in mouse hearts: critical role for expression level. Circulation. 2000; 101(14):1707-14. [PubMed: 10758054]

5. Iwase M, Bishop SP, Uechi M, Vatner DE, Shannon RP, Kudej RK, et al. Adverse effects of chronic endogenous sympathetic drive induced by cardiac Gs overexpression. Circ Res. 1996; 78(4):51724. [PubMed: 8635208]

6. Communal C, Singh K, Sawyer DB, Colucci WS. Opposing effects of [beta]1- and [beta]2adrenergic receptors on cardiac myocyte apoptosis: Role of a pertussis toxin-sensitive $\mathrm{G}$ protein. Circulation. 1999; 100(22):2210-2. [PubMed: 10577992]

7. Sabbah HN, Sharov VG, Gupta RC, Todor A, Singh V, Goldstein S. Chronic therapy with metoprolol attenuates cardiomyocyte apoptosis in dogs with heart failure. J Am Coll Cardiol. 2000; 36(5):1698-705. [PubMed: 11079679]

8. Communal C, Singh K, Pimentel DR, Colucci WS. Norepinephrine stimulates apoptosis in adult rat ventricular myocytes by activation of the $\beta$-adrenergic pathway. Circulation. 1998; 98(13):1329-34. [PubMed: 9751683]

9. Communal C, Singh M, Menon B, Xie Z, Colucci WSKS. beta1 integrins expression in adult rat ventricular myocytes and its role in the regulation of -adrenergic receptor-stimulated apoptosis. $\mathrm{J}$ Cell Bioch. 2003; 89(2):381-8.

10. Ross RS, Borg TK. Integrins and the myocardium. Circ Res. 2001; 88(11):1112-9. [PubMed: 11397776]

11. Schaller MD. Biochemical signals and biological responses elicited by the focal adhesion kinase. Biochim Biophys Acta. 2001; 1540(1):1-21. [PubMed: 11476890]

12. Bloch W, Forsberg E, Lentini S, Brakebusch C, Martin K, Krell HW, et al. beta 1 integrin is essential for teratoma growth and angiogenesis. J Cell Biol. 1997; 139(1):265-78. [PubMed: 9314545]

13. 1lic, Dk; Furuta, Y.; Kanazawa, S.; Takeda, N.; Sobue, K.; Nakatsuji, N., et al. Reduced cell motility and enhanced focal adhesion contact formation in cells from FAK-deficient mice. Nature. 1995; 377(6549):539-44. [PubMed: 7566154]

14. Shai S-Y, Harpf AE, Babbitt CJ, Jordan MC, Fishbein MC, Chen J, et al. Cardiac myocyte-specific excision of the \{beta\}1 integrin gene results in myocardial fibrosis and cardiac failure. Circ Res. 2002; 90(4):458-64. [PubMed: 11884376]

15. Peng X, Kraus MS, Wei H, Shen T-L, Pariaut R, Alcaraz A, et al. Inactivation of focal adhesion kinase in cardiomyocytes promotes eccentric cardiac hypertrophy and fibrosis in mice. J Clin Invest. 2006; 116(1):217-27. [PubMed: 16374517]

16. DiMichele LA, Doherty JT, Rojas M, Beggs HE, Reichardt LF, Mack CP, et al. Myocyte-restricted focal adhesion kinase deletion attenuates pressure overload-induced hypertrophy. Circ Res. 2006; 99(6):636-45. [PubMed: 16902179]

17. Franchini KG, Torsoni AS, Soares PHA, Saad MJA. Early activation of the multicomponent signaling complex associated with focal adhesion kinase induced by pressure overload in the rat heart. Circ Res. 2000; 87(7):558-65. [PubMed: 11009560]

18. Torsoni AS, Constancio SS, Nadruz W Jr, Hanks SK, Franchini KG. Focal adhesion kinase is activated and mediates the early hypertrophic response to stretch in cardiac myocytes. Circ Res. 2003; 93(2):140-7. [PubMed: 12805241]

19. Sabri A, Rafiq K, Seqqat R, Kolpakov MA, Dillon R, Dell'italia LJ. Sympathetic activation causes focal adhesion signaling alteration in early compensated volume overload attributable to isolated mitral regurgitation in the dog. Circ Res. 2008; 102(9):1127-36. [PubMed: 18356543]

20. Kolpakov MA, Seqqat R, Rafiq K, Xi H, Margulies KB, Libonati JR, et al. Pleiotropic effects of neutrophils on myocyte apoptosis and left ventricular remodeling during early volume overload. $\mathrm{J}$ Mol Cell Cardiol. 2009; 47(5):634-45. [PubMed: 19716828] 
21. Tallaj J, Wei C-C, Hankes GH, Holland M, Rynders P, Dillon AR, et al. \{beta\}1-adrenergic receptor blockade attenuates angiotensin II-mediated catecholamine release into the cardiac interstitium in mitral regurgitation. Circulation. 2003; 108(2):225-30. [PubMed: 12847066]

22. Tsutsui H, Spinale FG, Nagatsu M, Schmid PG, Ishihara K, DeFreyte G, et al. Effects of chronic [beta]-adrenergic blockade on the left ventricular and cardiocyte abnormalities of chronic canine mitral regurgitation. J Clin Invest. 1994; 93(6):2639-48. [PubMed: 7911128]

23. Rafiq K, Kolpakov MA, Abdelfettah M, Streblow DN, Hassid A, Dell'Italia LJ, et al. Role of protein-tyrosine phosphatase SHP2 in focal adhesion kinase down-regulation during neutrophil cathepsin G-induced cardiomyocytes anoikis. J Biol Chem. 2006; 281(28):19781-92. [PubMed: 16690621]

24. Gu J, Tamura M, Yamada KM. Tumor suppressor PTEN inhibits integrin- and growth factormediated mitogen-activated protein (MAP) kinase signaling pathways. J Cell Biol. 1998; 143(5): 1375-83. [PubMed: 9832564]

25. Communal C, Colucci WS, Singh K. p38 mitogen-activated protein kinase pathway protects adult rat ventricular myocytes against beta -adrenergic receptor-stimulated apoptosis. Evidence for Gidependent activation. J Biol Chem. 2000; 275(25):19395-400. [PubMed: 10770956]

26. Remondino A, Kwon SH, Communal C, Pimentel DR, Sawyer DB, Singh K, et al. \{beta\}adrenergic receptor-stimulated apoptosis in cardiac myocytes is mediated by reactive oxygen species/c-Jun NH2-terminal kinase-dependent activation of the mitochondrial pathway. Circ Res. 2003; 92(2):136-8. [PubMed: 12574140]

27. Melendez J, Welch S, Schaefer E, Moravec CS, Avraham S, Avraham H, et al. Activation of pyk2/ related focal adhesion tyrosine kinase and focal adhesion kinase in cardiac remodeling. $\mathrm{J}$ Biol Chem. 2002; 277(47):45203-10. [PubMed: 12228222]

28. Kubo H, Margulies KB, Piacentino V, Gaughan JP, Houser SR. Patients with end-stage congestive heart failure treated with $\beta$-adrenergic receptor antagonists have improved ventricular myocyte calcium regulatory protein abundance. Circulation. 2001; 104(9):1012-8. [PubMed: 11524394]

29. Swaney JS, Patel HH, Yokoyama U, Head BP, Roth DM, Insel PA. Focal adhesions in (myo)fibroblasts scaffold adenylyl cyclase with phosphorylated caveolin. J Biol Chem. 2006; 281(25):17173-9. [PubMed: 16618703]

30. Canetti C, Serezani CH, Atrasz RG, White ES, Aronoff DM, Peters-Golden M. Activation of phosphatase and tensin homolog on chromosome 10 mediates the inhibition of $F \hat{\mathrm{I}}^{3} \mathrm{R}$ phagocytosis by prostaglandin E2 in alveolar macrophages. J Immunol. 2007; 179(12):8350-6. [PubMed: 18056380]

31. Schwartzbauer G, Robbins J. The tumor suppressor gene PTEN can regulate cardiac hypertrophy and survival. J Biol Chem. 2001; 276(38):35786-93. [PubMed: 11448956]

32. Crackower MA, Oudit GY, Kozieradzki I, Sarao R, Sun H, Sasaki T, et al. Regulation of myocardial contractility and cell size by distinct PI3K-PTEN signaling pathways. Cell. 2002; 110(6):737-49. [PubMed: 12297047]

33. Oudit GY, Kassiri Z, Zhou J, Liu QC, Liu PP, Backx PH, et al. Loss of PTEN attenuates the development of pathological hypertrophy and heart failure in response to biomechanical stress. Cardiovasc Res. 2008; 78(3):505-14. [PubMed: 18281373]

34. Zhu W-Z, Wang S-Q, Chakir K, Yang D, Zhang T, Brown JH, et al. Linkage of \{beta $\}$ 1-adrenergic stimulation to apoptotic heart cell death through protein kinase A-independent activation of $\mathrm{Ca} 2+$ / calmodulin kinase II. J Clin Invest. 2003; 111(5):617-25. [PubMed: 12618516]

35. Taylor JM, Rovin JD, Parsons JT. A role for focal adhesion kinase in phenylephrine-induced hypertrophy of rat ventricular cardiomyocytes. J Biol Chem. 2000; 275(25):19250-7. [PubMed: 10749882]

36. Heidkamp MC, Bayer AL, Kalina JA, Eble DM, Samarel AM. GFP-FRNK disrupts focal adhesions and induces anoikis in neonatal rat ventricular myocytes. Circ Res. 2002; 90(12):12829. [PubMed: 12089066]

37. Bayer AL, Heidkamp MC, Patel N, Porter MJ, Engman SJ, Samarel AM. PYK2 expression and phosphorylation increases in pressure overload-induced left ventricular hypertrophy. Am J Physiol Heart Circ Physiol. 2002; 283(2):H695-706. [PubMed: 12124218] 


\section{Highlights}

- Acute VO induces extracellular matrix loss and FA signaling alterations

- $\quad \beta_{1}$-AR blockade attenuates VO-induced FA signaling alterations and myocyte apoptosis

- $\quad$ PTEN signaling mediates $\beta_{1}$-AR-induced FA signaling alterations and myocyte apoptosis 
A


B

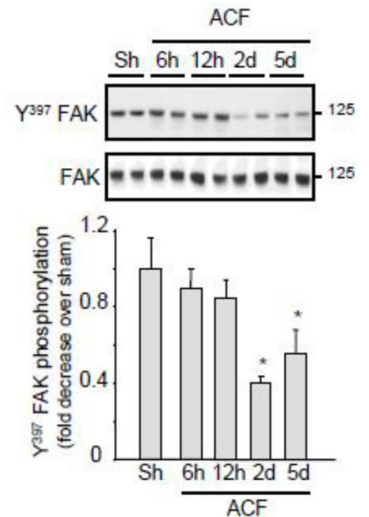

C
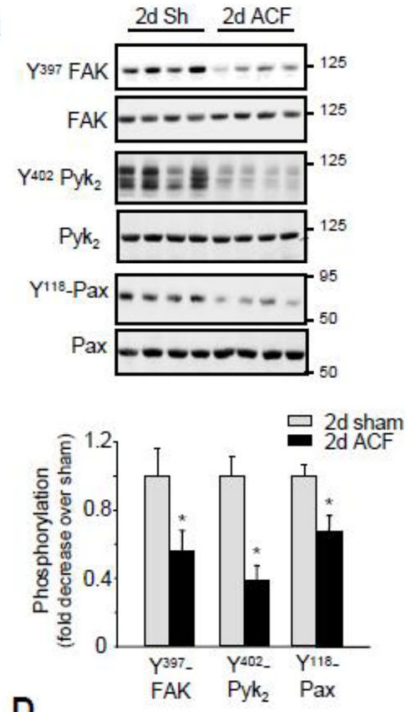

D

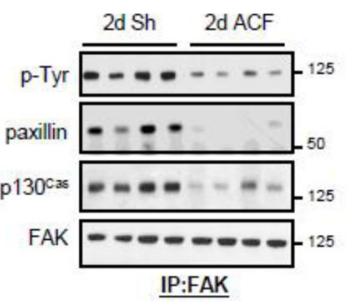

Figure 1. Acute ACF induces FA signaling downregulation

(A) Top, Representative picrosirius red staining.(Bar $=40 \mu \mathrm{M})$ Bottom, Interstitial collagen accumulation as determined by morphometric analysis. (B-C) LV extracts from control and ACF operated rats were assessed for immunoblot analysis. Top, representative immunoblots (with each lane from a single gel exposed for the same duration). Bottom, fold induction, $\mathrm{n}=6$ each group, $* P<0.05$ vs. sham. (D) LV lysates from sham or ACF animals were immunoprecipitated (IP) with anti-FAK antibodies and immunoblotted with antiphosphotyrosine, $\mathrm{p} 130^{\mathrm{Cas}}$, paxillin or FAK antibodies. 
A

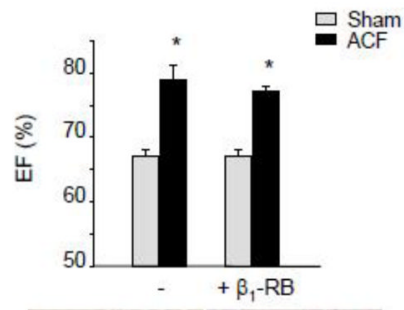

C

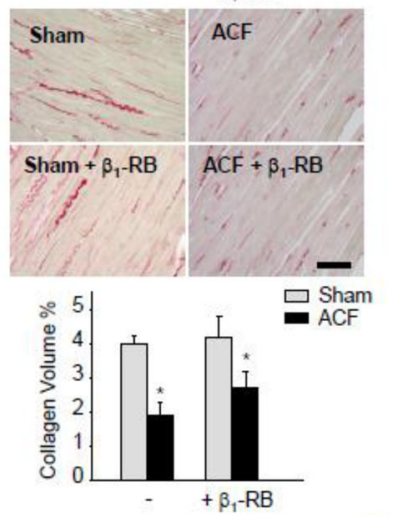

E
B

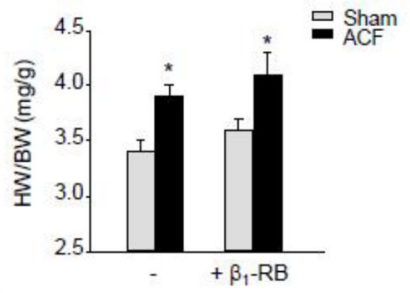

D


$\mathbf{F}$

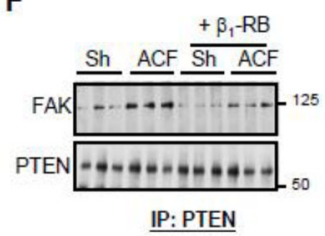

Figure 2. $\beta_{1}$-RB attenuates FA signaling downregulation induced after acute ACF Sham (Sh) and ACF animals were treated with vehicle or $\beta_{1}$-RB for 2-days. (A) Ejection fraction. (B) Heart weight to body weight ratio (HW/BW). (C) Top: Picrosirius red stained LV sections. (Bar $=40 \mu \mathrm{M})$ Bottom: Interstitial collagen volume quantification. Values are mean \pm SEM. $* P<0.05$ vs. sham ( $n=6$ for each experimental group) (D-E) Representative immunoblot showing accumulation of $\mathrm{Y}^{397}$-FAK and FAK (D) or $\mathrm{Y}^{580}-\mathrm{SHP}_{2}, \mathrm{SHP}_{2}$, nonphospho (np)-PTEN, and PTEN (E). Top, representative autoradiograms (with each lane from a single gel exposed for the same duration). Bottom, fold induction. $\mathrm{n}=6$ each group, $* P<0.05$ vs. sham, $\dagger P<0.05$ vs. ACF. (F) PTEN immunoprecipitates (IP) were immunoblotted with anti-FAK or -PTEN antibodies. 
A

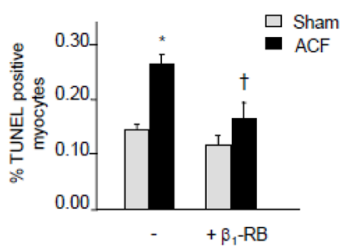

C

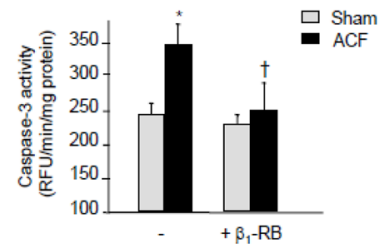

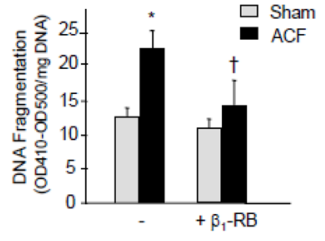

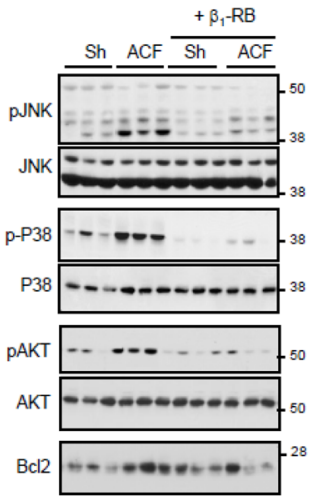

Figure 3. $\beta_{1}-R B$ attenuates myocyte apoptosis induced after acute ACF

(A) Number of TUNEL-positive myocytes was expressed as a percentage of total nuclei detected by DAPI staining. (B-C) LV homogenates from shams or animals subjected to $\mathrm{ACF}$ treated with vehicle or $\beta_{1}-\mathrm{RB}$ were assayed for DNA fragmentation assay using antihistone antibody ELISA (B) or for caspase-3 activity using specific fluorogenic substrate (C). Results are expressed as relative $\mathrm{OD}_{410}-\mathrm{OD}_{500} / \mathrm{mg}$ protein (B) or as relative fluorescence unit (RFU) $/ \mathrm{min} / \mathrm{mg}$ protein $(\mathrm{C})$. Values are mean \pm SEM. $* P<0.05$ vs. sham, $\dagger P<0.05$ vs. ACF. (D) Representative immunoblots in sham and ACF operated animals treated with vehicle or $\beta_{1}-\mathrm{RB}$. 
A

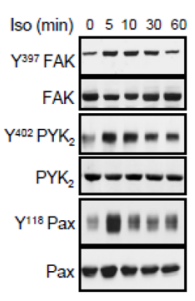

Pax $00-0-$

\section{C}

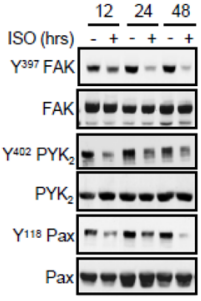

E

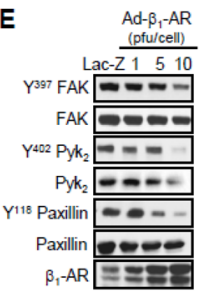

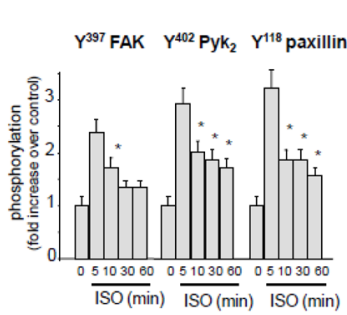
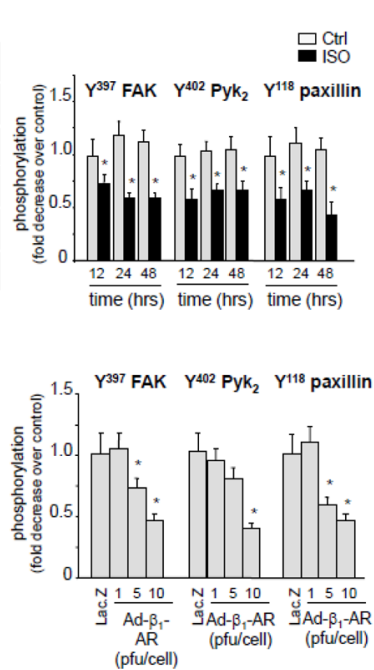

B

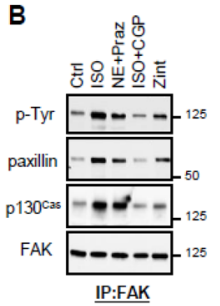

D

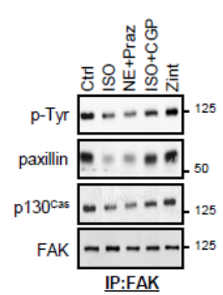

$\mathbf{F}$
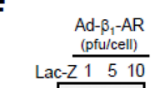

p-Tyr $-\cdots-125$

paxilin $=-\Delta-{ }_{-50}$

p130css $=-125$

FAK $=-125$

IP:FAK

Figure 4. Effect of acute versus chronic stimulation of $\boldsymbol{\beta}_{\mathbf{1}}$-ARs on FA signaling (A and C) Lysates from NRCMs untreated or treated with $10 \mu \mathrm{mol} / \mathrm{L}$ isoproterenol (ISO) for the indicated time were assessed for immunoblot analysis. (B and D) NRCMs were pretreated with $1 \mu \mathrm{mol} / \mathrm{L}$ prazosin (Praz) or $1 \mu \mathrm{mol} / \mathrm{L}$ CGP20712A (CGP) for 15-min prior to stimulation with $10 \mu \mathrm{mol} / \mathrm{L}$ ISO, $10 \mu \mathrm{mol} / \mathrm{L}$ norepinephrine $(\mathrm{NE})$, or $100 \mathrm{nmol} / \mathrm{L}$ zinterol (Zint) for 5-min (B) or 24-hrs (D). Cell lysates were immunoprecipitated with anti-FAK antibodies and then immunoblotted with anti-phosphotyrosine (P-Tyr), p130 ${ }^{\mathrm{Cas}}$, paxillin or FAK antibodies. (E-F) NRCMs were infected with Lac-Z (10 pfu/cell) or $\beta_{1}$-AR adenoviruses at the indicated titration and assayed for immunoblot analysis (E) or for immunoprecipitation assay using anti-FAK antibodies (F). Left: representative immunoblots. Right: Quantification of experiments expressed as mean \pm SE from three separate cultures. *P 0.05 vs. control. 

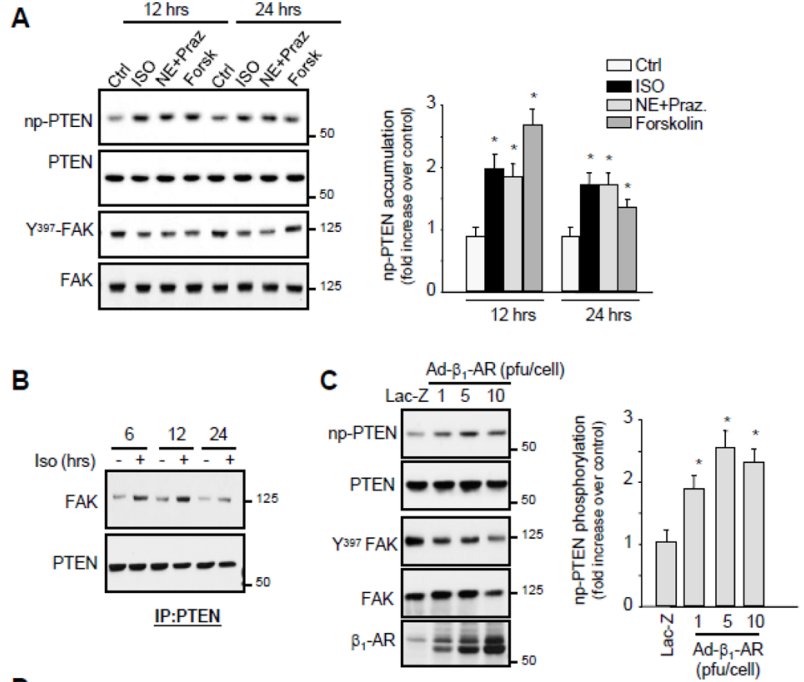

D
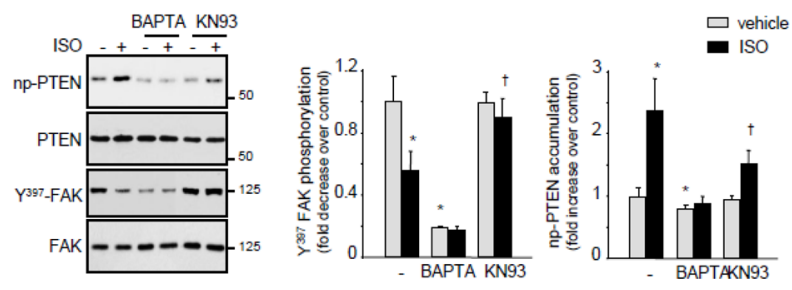

Figure 5. Chronic stimulation of $\beta_{1}$-ARs induces PTEN activation

(A) NRCMs were treated with isoproterenol (ISO), norepinephrine in presence of prazosin (NE+Praz.) and $1 \mu \mathrm{mol} / \mathrm{L}$ forskolin (Forsk) for 24-hrs and processed for immunoblot analysis. (B) PTEN immunoprecipitates from NRCMs untreated or treated with ISO for the indicated time were assayed for FAK and PTEN immunoblotting. (C) NRCMs were infected with Lac-Z (10 pfu/cell) or $\beta_{1}$-AR adenoviruses at the indicated titration and were assayed for immunoblot analysis. (D) NRCMs were pretreated with $5 \mu \mathrm{mol} / \mathrm{L}$ BAPTA-AM or 5 $\mu \mathrm{mol} / \mathrm{L}$ KN93 for 45-min prior to ISO treatment for 12- or 24-hrs and then assessed for immunoblot analysis. Left: Representative immunoblots. Right: Quantification of experiments expressed as mean $\pm \mathrm{SE}$ from three separate cultures. *P 0.05 vs. control; $\dagger \mathrm{P}$ 0.05 vs. ISO treated cells. 


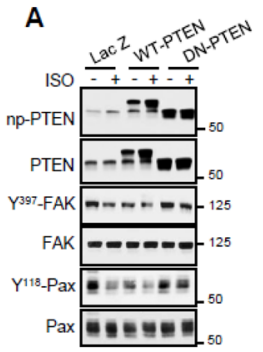

B

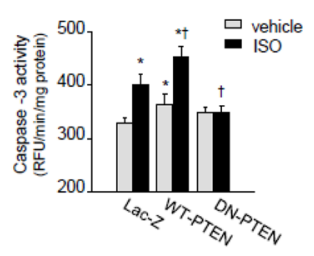

D

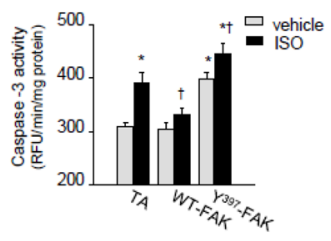

C

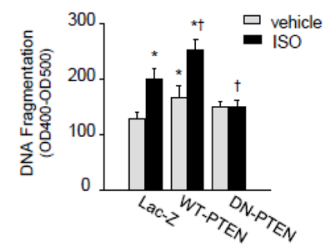

E

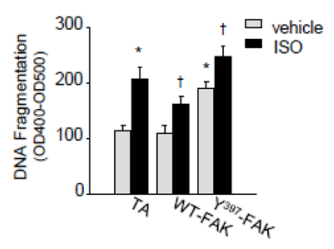

Figure 6. PTEN mediates FA signaling alteration and myocyte apoptosis induced by $\boldsymbol{\beta}_{\mathbf{1}}$-ARs NRCMs were infected with adenoviruses expressing Lac-Z (20 pfu/cell), WT-PTEN (20 pfu/cell), DN-PTEN (20 pfu/cell), transactivator (TA) (10 pfu/cell), WT-FAK (10 pfu/cell), or $\mathrm{Y}^{397}$ FAK mutant (10 pfu/cell) for 48-hrs. (A) Infected cells were treated with ISO for 24-hrs and then assessed for immunoblot analysis. Left: Representative immunoblots. Right: Quantification of experiments expressed as mean \pm SE from three separate cultures. *P 0.05 vs. Lac-Z control; $†$ P 0.05 vs. ISO treated Lac.Z infected cells. (B-E) Infected NRCMs were untreated or treated with ISO for 72-hrs. Lysates were processed for caspase-3 activity (B and $\mathrm{D}$ ) or DNA fragmentation assay (C and E). Results are for triplicate determinations from a single experiment (mean $\pm \mathrm{SE}$ ). $* \mathrm{P} 0.05$ vs. Lac-Z control; $\dagger \mathrm{P} 0.05$ vs. ISO treated cells. 


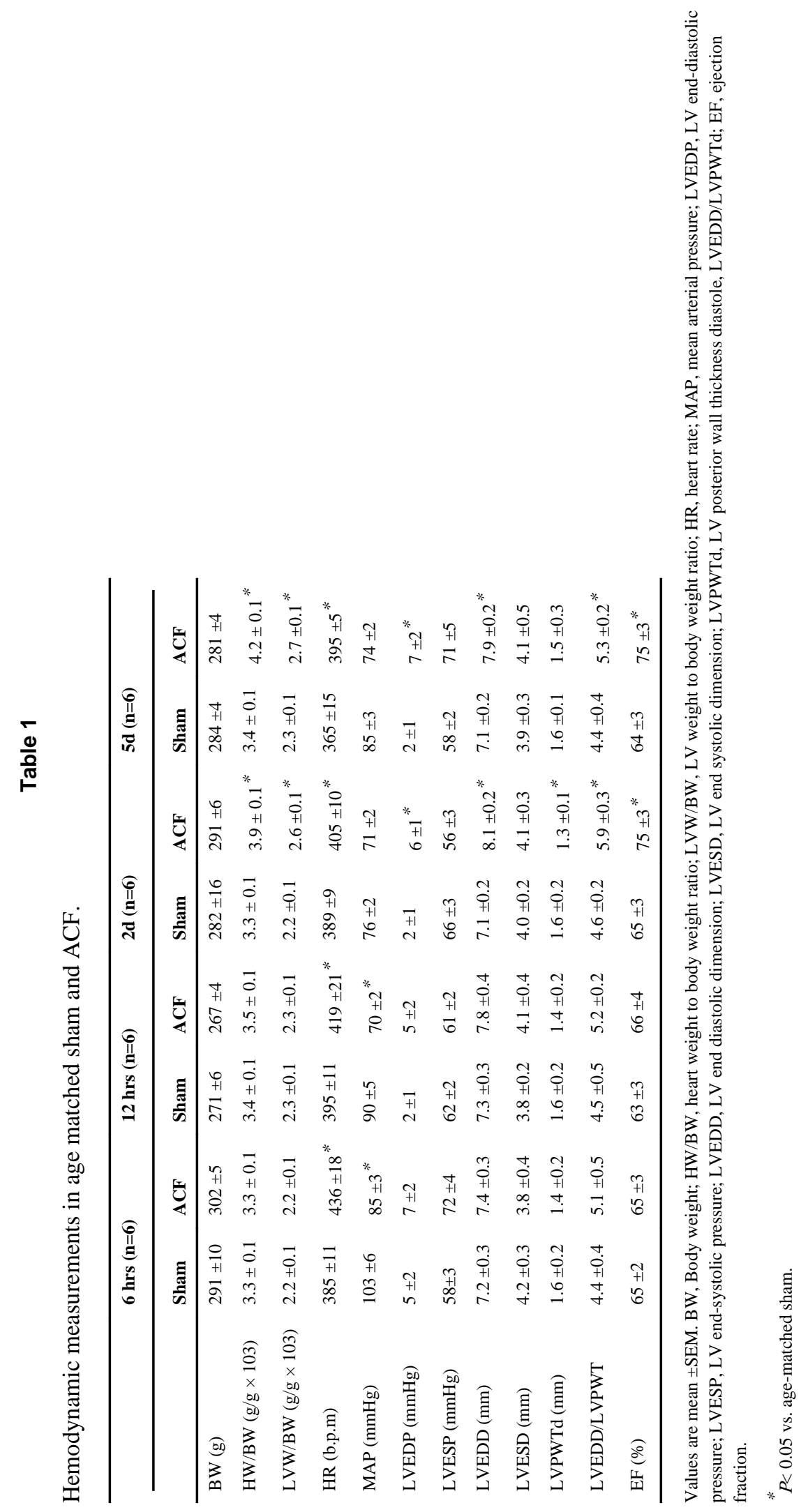

J Mol Cell Cardiol. Author manuscript; available in PMC 2013 November 14. 